\title{
Aplicação dos modelos lineares generalizados para estimativa do crescimento em altura
}

\author{
Andre Felipe Hess ${ }^{1 *}$, Lucas Dalmolin Cianorschi ${ }^{1}$, Raul Silvestre ${ }^{1}$, Rafael Scariot ${ }^{1}$, Pollyni Ricken ${ }^{1}$ \\ ${ }^{1}$ Universidade do Estado de Santa Catarina, Av. Luiz de Camões, 2090, CEP 88520-000, Lages, SC, Brasil
}

*Autor correspondente:

hessandre@yahoo.com.br

Termos para indexação:

Análise de tronco

Predição de estimativas

Acurácia do modelo

Index terms:

Stem analysis

Prediction estimates

Model accuracy

Histórico do artigo:

Recebido em 24/10/2013

Aprovado em 02/12/2015

Publicado em 31/12/2015

doi: $10.4336 / 2015 . p f b .35 .84 .604$

\begin{abstract}
Resumo - A análise do crescimento em altura é de extrema importância na área florestal, pois expressa a capacidade produtiva do local. Seu uso está associado ao ajuste, com menor erro, dos modelos para gerar estimativas que permitam a inferência com precisão e confiabilidade. O presente trabalho analisou o emprego dos modelos lineares generalizados na predição do crescimento em altura de Pinus taeda L. em função da idade e diâmetro a $1,30 \mathrm{~m}$ de altura em povoamentos no planalto catarinense. Os dados utilizados foram obtidos de análise de tronco completa de 25 árvores com oito anos, distribuídas em classes de diâmetro provenientes de Lages, SC. Os dados foram trabalhados na sua forma original sem a transformação das variáveis. O modelo com distribuição Gama e função de ligação identidade foi o que apresentou melhor ajuste, com desempenho superior nos critérios desvio $(1,21)$, Akaike $(255,39)$ e homogeneização dos resíduos, mostrando potencial para gerar estimativas da variável.
\end{abstract}

\section{Application of generalized linear models to estimate height growth}

\section{Introdução}

A produção e o estudo do crescimento dos parâmetros dendrométricos podem ser quantificados com o uso de funções florestais, visando atender os diferentes objetivos e concepções sobre seu desenvolvimento e sobre a organização da estruturação da produção florestal. Segundo Pretzsch (2009), o uso de modelos de crescimento florestal fornece um registro da evolução do conhecimento sobre as funções e estrutura da floresta, bem como favorecem o uso de métodos sofisticados para gerar as predições do crescimento em longo prazo.

O ajuste de uma função para descrever o crescimento pode ser realizado por meio de uma grande variedade de modelos matemáticos lineares e/ou não lineares, com base em determinadas distribuições de probabilidades. Os modelos não lineares, por exemplo, o de crescimento proposto por Richards (1959), apresentavam dificuldades para o ajuste devido à complexidade e à quantidade de cálculo interativo, mas se popularizaram atualmente 
com a implementação dos algoritmos em softwares computacionais específicos e avançados.

Analisar e descrever a influência que uma ou mais variáveis preditoras medidas em uma árvore ou em uma massa delas, requer a utilização de modelos matemáticos de regressão. Estes modelos são, sobretudo, sistemas de equações matemáticas que permitem a predição do crescimento e a produção de um povoamento, mediante duas características importantes: precisão e flexibilidade (Tomé, 1991), sendo a distribuição normal usada para modelos com dados contínuos e que tem uma distribuição simétrica (Dobson, 2002).

O problema na modelagem de grande parte dos dados, em várias áreas do conhecimento, são as características da heterocedasticidade e a autocorrelação, pois violam as pressuposições básicas da teoria dos modelos lineares e não lineares. Tais pressuposições consideram que a variável dependente ou o erro usualmente assumem uma distribuição normal (Nelder \& Wedderburn, 1972) ou que a frequência dos resíduos deve seguir uma distribuição normal (Schneider et al., 2009). Ainda segundo, Nelder \& Wedderburn (1972), técnicas desenvolvidas para dados com distribuição não normal incluem análise probit, onde uma variável binomial tem um parâmetro relacionado assumindo-se uma distribuição de tolerância, e tabelas de contingência, onde a distribuição é multidimensional e a parte sistemática do modelo é multiplicativa.

Quando a heterogeneidade da variância está presente, as observações não possuem distribuição idêntica e quando existe autocorrelação, as observações não são independentes. Dependendo da característica da base de dados, ambas as violações podem estar presentes (Calegario et al., 2005).

Durante muitos anos os modelos normais lineares foram utilizados na tentativa de descrever a maioria dos fenômenos aleatórios. Mesmo quando o fenômeno sob estudo não apresentava uma resposta para a qual fosse razoável a suposição de normalidade, algum tipo de transformação era sugerida, a fim de alcançar a normalidade procurada (Paula, 2013).

Assim, Nelder \& Wedderburn (1972) propuseram os modelos lineares generalizados (MLGs). A ideia básica consistiu em abrir o leque das opções para a distribuição da variável resposta, permitindo que a mesma pertença à família exponencial de distribuições, bem como dar maior flexibilidade para a relação funcional entre a média da variável resposta e o preditor linear $\eta$ (Paula,
2013). Este modelo (MLG) é definido em termos de um conjunto de variáveis independentes aleatórias, cada uma com distribuição para a família exponencial (Dobson, 2002).

O objetivo desse estudo foi analisar a qualidade do ajuste de um modelo matemático para o crescimento em altura em função das covariáveis diâmetro a $1,30 \mathrm{~m}$ do solo (DAP) e idade de árvores de Pinus taeda, utilizandose os modelos lineares generalizados na construção da equação que descreva com confiabilidade estatística a predição da variável para os demais indivíduos do povoamento. A finalidade é a obtenção de modelos sem transformação da variável e que gerem ajuste com precisão.

\section{Material e métodos}

\section{Base de dados e sítio de estudo}

A região do estudo é classificada, segundo Köeppen, como clima $\mathrm{Cfb}$ temperado constantemente úmido, com verão sem estação seca. A temperatura média anual varia de $13,8{ }^{\circ} \mathrm{C}$ a $15,8{ }^{\circ} \mathrm{C}$. A precipitação pluviométrica total anual pode variar de $1.360 \mathrm{~mm}$ a $1.600 \mathrm{~mm}$ e a umidade relativa do ar de $80 \%$ a $83 \%$ (Epagri, 2002).

Foi realizado inventário florestal no povoamento, por amostragem aleatória simples, com unidades amostrais temporárias de $20 \mathrm{~m} \times 20 \mathrm{~m}$, para obtenção das variáveis dendrométricas e estrutura do povoamento.

Foram derrubadas e seccionadas 25 árvores amostra de Pinus taeda com oito anos para análise de tronco (ANATRO) completa. As árvores foram obtidas em plantio de 15 ha em espaçamento de 2,5 $\mathrm{m} \mathrm{x} \mathrm{2,5} \mathrm{m,} \mathrm{em}$ Lages, SC, localizado a $27^{\circ} 50^{\prime} 49.66^{\prime \prime} \mathrm{S}$ e $50^{\circ} 22^{\prime} 13.90^{\prime}$ W, em altitude de $952 \mathrm{~m}$.

As árvores amostra foram divididas em três classes de DAP, sendo respectivamente, classe 1 , de $14 \mathrm{~cm}$ a $16,9 \mathrm{~cm}$, classe 2 , de $17 \mathrm{~cm}$ a $19,9 \mathrm{~cm}$ e classe $3 \mathrm{de}$ $20 \mathrm{~cm}$ a $22,9 \mathrm{~cm}$. A suficiência amostral foi calculada com base no equacionamento para população finita (Sanquetta, 2009).

A metodologia da ANATRO consistiu da retirada de discos com espessura de $3 \mathrm{~cm}$ a $5 \mathrm{~cm}$ para contagem dos anéis de crescimento, sendo retirados os discos nas posições de $0,10 \mathrm{~cm}, 1,30 \mathrm{~m}$ e a cada $2 \mathrm{~m}$ até a atingir $\mathrm{o}$ ápice do fuste, com posterior secagem (em ambiente natural), preparo (lixamento e marcação dos anéis). 
Os anéis de crescimento foram medidos em mesa de mensuração Lintab 6,0 (Rinntech, 2010).

Após processamento das medições, obteve-se um conjunto de dados com 153 observações de altura total e DAP por idade para as três classes diamétricas. Os ajustes dos modelos foram realizados no Sistema de Analise Estatístico SAS V. 9.1 (SAS Institute, 2004).

\section{Ajuste dos dados}

Inicialmente, os dados foram analisados para verificação do cumprimento dos condicionantes para ajuste de regressão, normalidade, independência e homogeneidade da variância. Contudo, frente ao não atendimento dos condicionantes, verificado pelos testes de Anderson-Darling, Durbin-Watson e de White optouse pelo não emprego de análise de regressão linear (modelo linear normal), mas sim pelo procedimento do ajuste com emprego dos modelos lineares generalizados. Essa opção foi estabelecida pelo critério de não adoção de transformação da variável dependente, evitandose a formação de viés em que o intercepto é afetado, indicando a necessidade de correção, bem como evitar problemas de discrepância logarítmica.

O modelo testado no ajuste foi $\mathrm{y}=\Phi_{0}+\Phi_{1} * d+\Phi_{2}{ }^{*} t+\varepsilon$, onde: y: variável resposta, Фs: coeficientes do modelo; $d$ : diâmetro a altura do peito; $t$ : idade e $\varepsilon$ : erro associado. O modelo foi ajustado nos componentes aleatórios, Normal, Poisson e Gama e funções de ligação identidade $(\mu)$ e logarítmica $\ln (\mu)$.

\section{Modelos lineares generalizados}

No presente estudo a situação experimental é expressa por uma variável aleatória $Y$ de interesse, denominada variável resposta ou dependente e um vector $\chi=\left(\chi_{1}, \ldots, \chi_{\kappa}\right)$ de variáveis explicativas designadas por covariáveis ou variáveis independentes, que se acredita explicar parte da variabilidade inerente a $Y$ (Turkman \& Silva, 2000).

Os modelos lineares generalizados são uma extensão do modelo linear clássico $Y=Z \beta+\varepsilon$, onde $Z$ é uma matriz de dimensão $\eta \times \mathrm{p}$ de especificação de modelo (em geral a matriz de covariáveis $\mathrm{X}$ com um primeiro vetor unitário), associada a um vetor $\beta=\left(\beta_{1}, \ldots, \beta_{p}\right)^{T}$ de parâmetros e $\varepsilon$ é um vetor de erros aleatórios com distribuição $\mathrm{N}_{\mathrm{n}}\left(0, \sigma^{2}, \mathrm{I}\right)$.

Segundo Turkman \& Silva (2000), a escolha da função de ligação depende do tipo de resposta e do estudo em particular. Algumas das principais funções de ligação são: identidade, função quadrática, raiz quadrada, expoente, logística, logarítmica entre outras.

\section{Qualidade do ajuste dos MLG}

A qualidade do ajuste de um MLG é avaliada através da função desvio:

$$
D^{*}(v ; \widehat{\mu})=\phi D(v ; \widehat{\mu})=2\{L(v ; v)-L(\widehat{\mu} ; v)\}
$$

A função desvio resulta em uma distância entre o logaritmo da função de verosimilhança do modelo saturado (com $\eta$ parâmetros) e do modelo de investigação (com $p$ parâmetros) avaliado na estimativa de máxima verosimilhança $\hat{\beta}$. Um valor pequeno para a função de desvio indica que, para um número menor de parâmetros se obtém um ajuste tão bom quanto o ajuste com o modelo saturado (Paula, 2013).

A Tabela 1 apresenta as expressões das funções desvio para os componentes aleatórios utilizados no ajuste do modelo por MLG neste trabalho.

Tabela 1. Expressões das funções desvios para os campos aleatórios da função de distribuição utilizados no ajuste dos dados.

\begin{tabular}{cc}
\hline Modelo & Equação \\
\hline Normal & $\Sigma_{i}\left(y_{i}-\widehat{\mu}_{i}\right)$ \\
Poisson & $2\left|\sum_{i} y_{i} \ln \frac{y_{i}}{\hat{\mu}_{i}}-\sum_{i}\left(y_{i}-\widehat{\mu}_{i}\right)\right|$ \\
Gama & $2 \sum_{i}\left\{-\ln \frac{y_{i}}{\widehat{\mu}_{i}}+\frac{y_{i}-\widehat{\mu}_{i}}{\widehat{\mu}_{i}}\right\}$ \\
\hline
\end{tabular}

A seleção de MLG também é possível utilizandose o critério de informação (AIC) de Akaike (1974), que se baseia na função log-verossimilhança, com a introdução de um fator de correção como modelo de penalização da complexidade do modelo (Nelder \& Wedderburn, 1972; Turkman \& Silva, 2000; Paula, 2013). A estatística $A I C$ é amplamente utilizada para selecionar o melhor entre os modelos paramétricos alternativos. O procedimento de seleção do modelo $A I C$ mínimo pode ser interpretado como uma maximização da entropia esperada (Akaike, 1981). A estatística correspondente é: $A I C=-2 \ell\left(\tilde{\beta}_{1}, 0, \phi\right)+2 r$, onde $r$ é função de log-verossimilhança esperada. Um valor baixo para o $A I C$ é considerado representativo de um melhor ajuste, tendo como objetivo a minimização desse valor. 


\section{Análise dos resíduos}

A distribuição da frequência dos resíduos nesse trabalho, à semelhança do resíduo para o modelo linear normal, definiu-se pelo resíduo de Pearson, calculado para cada um dos campos aleatórios dos MLG, como apresentado na Tabela 2 .

Tabela 2. Expressão dos resíduos padronizados e generalizados por Pearson.

\begin{tabular}{cc}
\hline MLG & $R_{I}^{P}$ \\
\hline Normal & $y_{i}-\hat{\mu}_{i}$ \\
Gama & $\frac{y_{i}-\hat{\mu}_{i}}{\hat{\mu}_{i}}$ \\
Poisson & $\frac{y_{i}-\hat{\mu}_{i}}{\hat{\hat{\mu}}^{1 / 2}}$ \\
\hline MLG = modelo linear generalizado; $\mathrm{R}=; \hat{\mu_{i}}$
\end{tabular}

Nos MLGs, segundo Cordeiro \& Lima Neto (2006), os resíduos são usados para explorar a adequação do modelo ajustado com respeito à escolha da função de variância, da função de ligação e de termos do preditor linear. Também são importantes para a identificação de outliers e medem a discrepância entre os valores observados $\mathrm{y}_{\mathrm{i}}$ e ajustados $\hat{\mu}_{i}$.

\section{Resultados e discussão}

A análise dos dados para o teste de normalidade apresentou estatística de Anderson-Darling de 2,37 e P-valor de $0,0005 \%$, rejeitando-se a hipótese de normalidade dos resíduos da altura com nível de confiança de $95 \%$, (Figura 1) do papel de probabilidade. O teste de Durbin-Watson (DW) acusou um valor de 0,52 , comprovando que os resíduos são dependentes. $\mathrm{O}$ teste de White acusou um valor de qui-quadrado igual a 21,5 ( $\mathrm{p}=0,0007)$, refletindo a heterogeneidade da variância, o que indica a necessidade de transformação ou ajuste por outra técnica de regressão (Schneider et al., 2009).

Os testes e a observação da Figura 1 demonstram inadequação ao modelo linear normal, dando suporte ao uso dos modelos lineares generalizados nas famílias de distribuição e função de ligação.

Na Tabela 3 podem ser verificados os critérios do ajuste para os MLG utilizando as funções de ligação

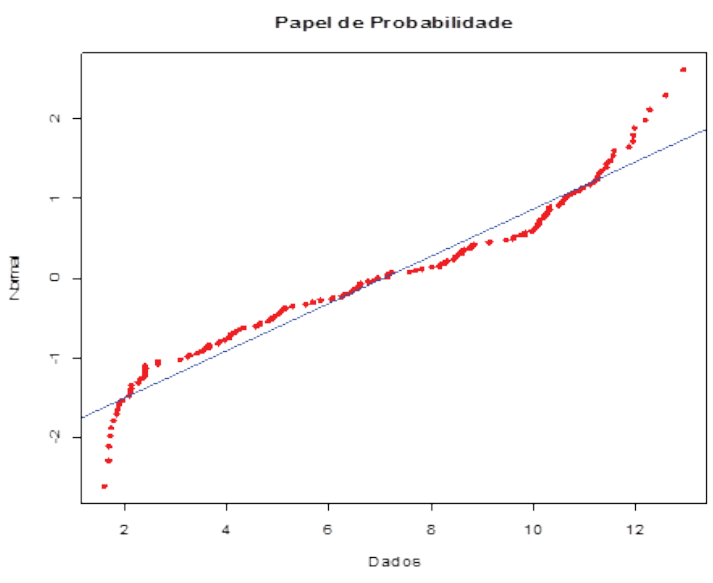

Figura 1. Papel de probabilidade para distribuição normal dos resíduos de altura das árvores.

identidade e logarítmica, e os valores dos critérios de desvio, o logarítmico da máxima verossimilhança, Akaike. A distribuição dos resíduos está apresentada na Figura 2.

Os MLG Gama- $\mu, \ln (\mu)$ e Normal- $\ln (\mu)$ tiveram todos os parâmetros com nível de significância nominal (valores $p$ ), inferiores a 0,0001 , indicando o efeito da idade e do diâmetro no crescimento em altura. O modelo Gama- $\ln (\mu)$ foi o que apresentou os menores valores para o erro padrão, gerando, segundo Calegario et al. (2005), intervalos de confiança de menor amplitude. Por sua vez, o modelo Poisson- $\mu$ foi o que apresentou os maiores valores para o erro padrão e o modelo Normal- $\mu$ (parâmetro intercepto) e Poisson- $\ln (\mu)\left(b_{1}{ }^{*} x\right.$ e $\left.b_{2}{ }^{*} x_{1}\right)$ tiveram significância superior a 0,0001 , indicando que o uso desses modelos não garante estimativas da altura com acurácia para as diferentes idades e diâmetros (Mendonça et al., 2015).

Contudo, o modelo Gama- $\mu$ foi o que apresentou melhores resultados quando considerados os critérios desvio $(1,21)$, AIC $(255,39)$ e resíduos homogeneizados (Figura 2), mostrando-se como um guia para avaliar a adequabilidade do modelo, contribuindo para redução da variabilidade não explicada da altura e, conforme Mendonça et al. (2015) estimando alturas com melhor acurácia, precisão e condizente às alturas reais. Além disso, o modelo apresenta vantagens, pois descarta tendências de transformação da variável advinda da discrepância logarítmica. 
Tabela 3. Valores dos parâmetros e qualidade do ajuste dos MLG para ajuste do crescimento de altura em função da idade e diâmetro.

\begin{tabular}{|c|c|c|c|c|c|c|}
\hline \multirow[t]{2}{*}{ MLG - FL } & \multirow{2}{*}{$\begin{array}{c}\text { Parâmetros } \\
\text { Valor }\end{array}$} & \multicolumn{2}{|c|}{ Estimativas } & \multicolumn{3}{|c|}{ Critério do ajuste } \\
\hline & & stEerro & Prob. $^{2}$ & D & Log-veros & AIC \\
\hline \multicolumn{7}{|l|}{ Gama- $\mu$} \\
\hline Intercepto & 0,5834 & 0,1088 & $<0,0001$ & & & \\
\hline $\mathrm{x}$ & 0,4538 & 0,0687 & $<0,0001$ & 1,2061 & $-123,6961$ & 255,3921 \\
\hline$x_{1}$ & 0,4699 & 0,0273 & $<0,0001$ & & & \\
\hline \multicolumn{7}{|l|}{$\operatorname{Gama-\operatorname {ln}(\mu )}$} \\
\hline Intercepto & 0,6904 & 0,0415 & $<0,0001$ & & & \\
\hline $\mathrm{x}$ & 0,0898 & 0,0206 & $<0,0001$ & 4,1767 & $-218,9673$ & 445,9346 \\
\hline$x_{1}$ & 0,0769 & 0,0086 & $<0,0001$ & & & \\
\hline \multicolumn{7}{|l|}{ Normal- $\mu$} \\
\hline Intercepto & 0,1522 & 0,1701 & 0,3709 & & & \\
\hline $\mathrm{x}$ & 0,7845 & 0,0847 & $<0,0001$ & 71,5481 & $-158,9524$ & 325,9048 \\
\hline$x_{1}$ & 0,3366 & 0,0345 & $<0,0001$ & & & \\
\hline \multicolumn{7}{|l|}{$\operatorname{Normal}-\ln (\mu)$} \\
\hline Intercepto & 0,9226 & 0,0419 & $<0,0001$ & & & \\
\hline $\mathrm{x}$ & 0,1171 & 0,0133 & $<0,0001$ & 155,0297 & $-218,1058$ & 444,2116 \\
\hline$x_{1}$ & 0,0413 & 0,0053 & $<0,0001$ & & & \\
\hline \multicolumn{7}{|l|}{ Poisson- $\mu$} \\
\hline Intercepto & 0,2841 & 0,5656 & 0,6155 & & & \\
\hline $\mathrm{x}$ & 0,6626 & 0,3388 & 0,0505 & 8,6275 & 1147,9204 & 577,8276 \\
\hline$x_{1}$ & 0,3889 & 0,1361 & 0,0043 & & & \\
\hline \multicolumn{7}{|l|}{ Poisson-ln $(\mu)$} \\
\hline Intercepto & 0,8015 & 0,1052 & $<0,0001$ & & & \\
\hline $\mathrm{x}$ & 0,1126 & 0,0407 & 0,0056 & 23,9495 & 1140,2594 & 593,1496 \\
\hline$x_{1}$ & 0,0538 & 0,0166 & 0,0012 & & & \\
\hline
\end{tabular}

Onde: MLG-FL: modelo linear generalizado, campo aleatório Gama, Normal, Poisson e função de ligação; $\mu$ : identidade, $\ln (\mu)$ : logarítmica; $\mathrm{x}$ : covariável idade; $\mathrm{x}_{1}$ : covariável diâmetro; stEerro: erro padrão; Prob. ${ }^{2}$ : probabilidade ${ }^{2}$ de Wald; $D$ : desvio; Log-veros: log-verossimilhança; AIC: critério de Akaike.

Portanto, o modelo Gama- $\mu$ possibilita estimar com melhor precisão a variável resposta altura, pois as variáveis diâmetro e idade afetam de forma significativa o crescimento em altura. Carvalho et al. (2011), para estudo da predição da área basal e volume para clones de eucalipto, também encontraram melhor ajuste com equação completa, usando como covariáveis o índice de sítio e o número de plantas por hectare. A distribuição Gama oferece grande potencial para a modelagem de dados contínuos que apresentam superdispersão e, portanto, heterogeneidade de variância, o que foi amplamente estudado por Gea-Izquierdo \& Cañellas (2009), Contreras et al. (2011), dentre outros.

$\mathrm{O}$ adequado ajuste do modelo Gama função de ligação identidade pode ser usado com eficiência para a estimativa da altura. $\mathrm{O}$ correto ajuste da variável altura (sem tendenciosidade) é de extrema importância, pois interfere de forma significativa na estimativa do volume. A necessidade de precisão é assim requerida, pois o volume é a base para o planejamento e ordenamento da produção no manejo florestal, avaliação do valor do povoamento e de rentabilidade florestal.

Verifica-se na Figura 2, que o modelo Gama- $\mu$ apresenta resíduos com média zero, variância desconhecida e homogeneização dos resíduos. Desta forma, o ajuste do modelo proporcionou que os erros tendessem a uma distribuição aleatória em torno de zero (Carvalho et al., 2011). O uso do modelo Gama resultou em um modelo que gera ajustes com melhor acurácia e que descreve a real relação entre as covariáveis e a variável dependente.

Os modelos Normal- $\mu$ e Poisson- $\mu$ apresentam heterocedasticidade do tipo funil crescente e os modelos Gama- $\ln (\mu)$, Normal- $\ln (\mu)$ e Poisson- $\ln (\mu)$ demonstram a necessidade de um termo quadrático no ajuste do modelo (Scolforo, 2005). O uso dos modelos Gama-ln( $\mu$ ), Normal- $\ln (\mu)$ e Poisson- $\ln (\mu)$ estimam em diâmetros e idades menores os valores de altura e, com o aumento do diâmetro e idade, valores superestimados para a variável. 


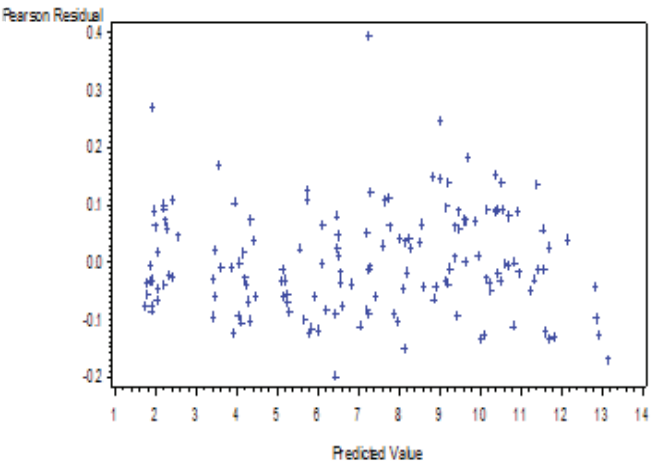

Gama- $\mu$

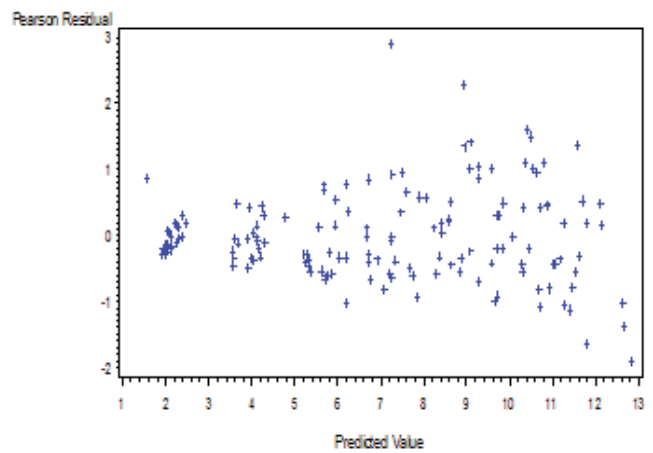

Normal- $\mu$

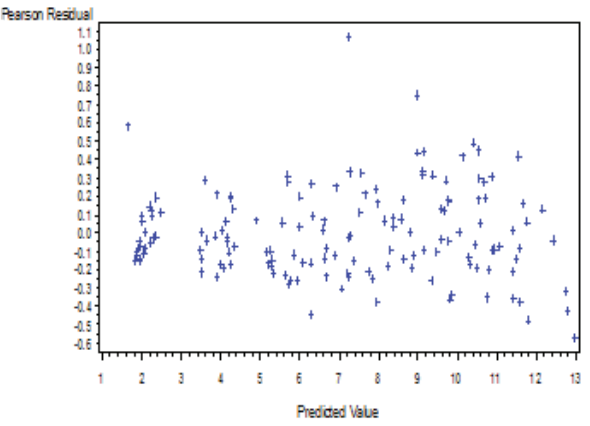

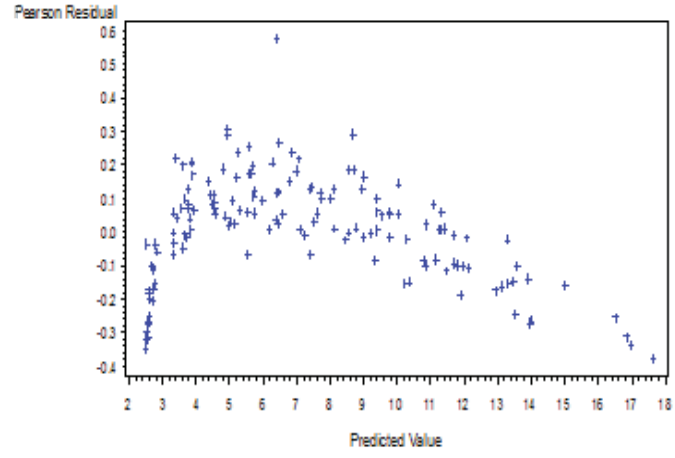

$\operatorname{Gama-\operatorname {ln}(\mu )}$

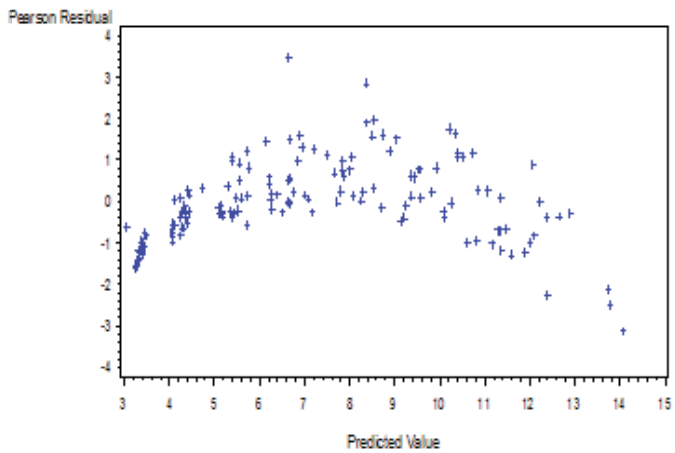

Normal- $\ln (\mu)$

Figura 2. Diagnóstico do resíduo para os MLG segundo campo aleatório e função de ligação.

Eixo x: valor estimado. Eixo y: resíduo de Pearson.

\section{Conclusões}

Os MLG ajustaram os dados de crescimento com precisão significativa no campo aleatório Gama e função de ligação identidade, sendo justificável o seu uso para gerar estimativas com acurácia, principalmente porque não necessitaram de transformação nas variáveis.

O sistema gera modelos precisos e com poucos parâmetros de interpretação, sendo conveniente pela não necessidade de um conjunto maior de dados e covariáveis adicionais ao modelo para melhora do ajuste. $\mathrm{O}$ ajuste do modelo Gama comprova sua eficiência para análise de dados não negativos e de natureza contínua e que apresentam variância crescente com a média.

O emprego de técnicas de ajuste de equações para gerar estimativas dendrométricas com precisão é 
importante para o desenvolvimento do manejo florestal sustentável.

\section{Referências}

AKAIKE, H. A new look at the statistical model identification. IEEE Transactions on Automatic Control, Boston, v. 19, n. 6, p. 716-723, Dec. 1974.

AKAIKE, H. Likelihood of a model and information criteria. Journal of Econometrics, Amsterdam, n. 16, p. 3-14, 1981. DOI: 10.1016/0304-4076(81)90071-3.

CAlEGARIO, N.; CALEGARIO, C. L. L.; MAESTRI, R.; DANIELS, R. Melhoria da qualidade de ajuste de modelos biométricos florestais pelo emprego da teoria dos modelos não lineares generalizados. Scientia Forestalis, Piracicaba, n. 69, p. 38-50, dez. 2005.

CARVALHO, S. de P. C.; CALEGARIO, N.; SILVA, F. F.; BORGES, L. A. C.; MENDONÇA, A. R. de; LIMA, M. P. de. Modelos não lineares generalizados aplicados na predição da área basal e volume de Eucalyptus clonal. Cerne, Lavras, v. 17, n. 4, p. 541-548, out./ dez. 2011. DOI: 10.1590/S0104-77602011000400013.

CORDEIRO, G. M.; LIMA NETO, E. A. Modelos paramétricos. Recife: Universidade Federal Rural de Pernambuco, Departamento de Estatística e Informática, 2006. 256 p.

CONTRERAS, M. A.; AFFLECK, D.; CHUNG, W. Evaluating tree competition indices as predictors of basal area increment in western Montana forests. Forest Ecology and Management, Amsterdam, v. 262, p. 1939-1949, 2011. DOI: 10.1016/j.foreco.2011.08.031.

DOBSON, J. A. An a introduction to generalized linear models. 2nd ed. Florida: Chapman \& Hall, 2002. 221 p.

EPAGRI. Dados e informações biofísicas da unidade de planejamento regional do planalto sul-catarinense - UPR 3. Florianópolis, 2002. $70 \mathrm{p}$.

GEA-IZQUIERDO, G.; CAÑELLAS, I. Analysis of Holm Oak intraspecific competition using Gama regression. Forest Science, Bethesda, v. 55, p. 310-322, 2009.
MENDONÇA, A. R. de; CARVALHO, S. de P. C. e; CALEGARIO, N. Modelos hipsométricos generalizados mistos na predição da altura de Eucalyptus sp. Cerne, Lavras, v. 21, n. 1, p. 107-115, 2015. DOI: 10.1590/01047760201521011191.

NELDER, J. A.; WEDDERBURN, R. W. M. Generalized linear models. Journal of the Royal Statistical Society, London, v. 135, n. 3, p. 370-384, 1972.

PAULA, G. A. Modelos de regressão com apoio computacional. São Paulo: Universidade de São Paulo, Instituto de Matemática e Estatística, [s.d.]. Disponível em: <http://www.ime.unicamp. br/ cnaber/Livro_MLG.pdf $>$. Acesso em: 1 maio 2013.

PRETZSCH, H. Forest dynamics, growth and yield. Berlin: Springer Verlag, 2009. 664 p.

RICHARDS, F. J. A Flexible growth function for empirical use. Journal of Experimental Botany, London, v. 10, n. 2, p. 290-301, 1959. DOI: $10.1093 / \mathrm{jxb} / 10.2 .290$.

RINNTECH. TSAP-WIN: time series analysis and presentation dendrochonology and related applications. Heidelberg, 2010.

SANQUETTA, C. R.; WATZLAWICK, L. F.; CÔRTE, A. P. D.; FERNANDES, L. de A. V.; SIQUEIRA, J. D. P. Inventários florestais: planejamento e execução. 2. ed. Curitiba, 2009. 316 p.

SAS INSTITUTE. The SAS system for Windows. Cary, NC, 2004 SCHNEIDER, P. R.; SCHNEIDER, P. R. P.; SOUZA, C. A. M. de. Análise de regressão aplicada à engenharia florestal. 2. ed. Santa Maria, RS: RS: Universidade Federal de Santa Maria, Faculdade de Ciências da Comunicação, 2009. 249 p.

SCOLFORO, J. R. S. Biometria florestal: parte I: modelos de regressão linear e não-linear, parte II: modelos para relação hipsométrica, volume, afilamento e peso de matéria seca. Lavras: Universidade Federal de Lavras, Fundação de Apoio ao Ensino, Pesquisa e Extensão, 2005. 325 p.

TOMÉ, M. Os modelos de produção florestal na gestão de povoamentos florestais. Revista Agros, v. 8, p. 32-36, 1991.

TURKMAN, M. A. A.; SILVA, G. L. Modelos lineares generalizados: da teoria à prática. 2000.153 p. Apostila do mini-curso ministrado no VIII Congresso Anual da Sociedade Portuguesa de Estatística, 2000, Lisboa. 
